\title{
A Rapid and Sensitive LC - MS/MS Assay for the Quantitation of Bromantane in Human Plasma
}

\author{
Igor I Miroshnichenko, Svetlana A Sergeeva, Angelina I Platova* and Lyudmila M Krasnykh
}

Mental Health Research Center of RAMS, Moscow, Russia

\begin{abstract}
The purpose of this study was to develop and validate a high-performance liquid chromatographic-tandem mass spectrometric (LC-MS/MS) method for analysis of the bromantane in human plasma. The analyte and Internal Standard (IS), selenox, were extracted from human plasma by solid-phase extraction and separated on a Zorbax SB-C18 column using methanol-0.2\% formic acid as mobile phase. Detection was performed using an atmospheric pressure chemical ionization source and mass spectrometric positive Multi-Reaction-Monitoring-Mode (+MRM) at an ion voltage of $+2000 \mathrm{~V}$. The assay was linear over the concentration range $1-500 \mathrm{ng} / \mathrm{mL}$ with the Lowest Limit of Quantification (LLOQ) of $1 \mathrm{ng} / \mathrm{mL}$. The method also afforded satisfactory results in terms of the sensitivity, specificity, precision (intra- and inter-day, $\mathrm{CV}<10 \%$ ), accuracy, recovery as well as the stability of the analyte under various conditions. The method can be applied to pharmacokinetic and toxicological studies.
\end{abstract}

Keywords: Bromantane; Liquid Chromatography-Mass Spectrometry (LC-MS/MS); APCI; Plasma

\section{Introduction}

Bromantane, N-(4-bromophenyl) adamantan-2-amine, is a drug with anxiolytic and immunostimulatory actions and elements of actoprotective activity [1-3]. Bromantane is not potentially addictive and does not exert effects of hypnosedation or neuromuscular relaxation. The use of the drug, in contrast to the action of a typical psycho stimulant, is not associated with the phenomenon of hyper stimulation and causes no consequences such as functional exhaustion of the body [4]. These properties of drug stipulated its using as a doping agent [5]. According to World Anti-Doping Agency classification, Bromantane belongs to S6; a class of prohibited substances [6].

The therapeutic action of Bromantane in patients with asthenic and anxiety-asthenic disorders is exhibited from first day of application, which is expressed by a marked reduction of asthenic symptoms, signs of emotional tension, and somatoautonomic manifestations; the drug works by restoring performance and enhancing the endurance of the body. Its mechanism of action is associated with the release of reinforcing dopamine from the presynaptic terminal [7], blockade of its reuptake, and enhancement of biosynthesis and induced tyrosine hydroxylase gene expression, as well as with a modulatory effect on the Benzodiazepine-GABA-receptor-chloride ion channel complex, upregulating the stress-induced decrease of benzodiazepine reception. Bromantane increases GABAergic transmission, reduces gene expression, and regulates the synthesis of GABA-mediators, which act as reuptake transmitters. Bromantane has low toxicity (LD50 in rats is higher than $10000 \mathrm{mg} / \mathrm{kg}$, exceeding the effective dose by 100 times).

Appropriate analytical method is an urgent need to studybromantane pharmacokinetic properties. The gas chromatography approach for content determination has been applied to a pharmacokinetic study of Bromantane [8]. However, this method was not quite appropriate for clinic pharmacokinetic studies of bromantane. LC/MS spectrometry based techniques are now the mainstay for such pharmacokinetic studies because of sensitivity, selectivity, speed and cost effectiveness [9]. Recently some papers devoted LC-MS/MS method for the quantitative estimation of amantadine (1-adamantylamine) [10] and memantine appeared in press $[11,12]$.

The most commonly used sample preparation methods for blood plasma samples are Liquid-Liquid Extraction (LLE) and Solid-Phase Extraction (SPE). SPE is growing in use, and it offers certain advantages over LLE, including better specificity, the ability to obtain cleaner samples, good reproducibility, and a substantial reduction in the volume of solvent required [13].

However, we do not find HPLC-MS/MS methods reported in the literature for quantification of bromantane in biological samples. This paper reports on a rapid and sensitive LC-MS/MS method for the determination of bromantane in human plasma using SOLA cartridges. The method employs using 9-phenyl-sym-octahydroselenoxanthene (selenox) as internal standard and is completed in a run time of $6 \mathrm{~min}$.

\section{Experimental}

\section{Chemicals and reagents}

Bromantane was kindly supplied by the Pharmacology Institute of RAMS, Moscow, 9-phenyl-sym-octahydroselenoxanthene synthesized by "Medbiopharm", Obninsk, and Kaluga region. Formic acid (Sigma Inc., USA), methanol and acetonitrile (Lab-Scan, Poland) were HPLCgrade. Deionized water was prepared from distilled water using Simplicity UV System (Millipore, USA). All other chemicals and solvents were analytical grade and used as received. The SOLA reversed phase extraction cartridges were purchased from Thermo Fisher Scientific Inc. (USA).

\section{LC-MS/MS conditions}

The HPLC-MS/MS system consisted of an Agilent 1200 series binary pump, an auto sampler connected to Agilent $6410-2 \mathrm{~K}$ Triple Quad LC-MS (Agilent Technologies, Palo Alto, CA, USA) using a atmospheric pressure chemical ionization (APCI). The instrument was interfaced to a computer running Agilent Mass Hunter B.01.04. Software.

*Corresponding author: Angelina I Platova, Mental Health Research Center of RAMS, Moscow, Russia, E-mail: Platova6283@mail.ru

Received January 20, 2013; Accepted February 21, 2013; Published February 25,2013

Citation: Miroshnichenko II, Sergeeva SA, Platova AI, Krasnykh LM (2013) A Rapid and Sensitive LC-MS/MS Assay for the Quantitation of Bromantane in Human Plasma. J Sports Med Doping Stud 3: 120. doi:10.4172/2161-0673.1000120

Copyright: () 2013 Miroshnichenko II, et al. This is an open-access article distributed under the terms of the Creative Commons Attribution License, which permits unrestricted use, distribution, and reproduction in any medium, provided the original author and source are credited. 
Bromantane and IS were separated a Zorbax SB-C18 column $(3.5 \mu \mathrm{m}, 100 \mathrm{~mm} \times 4.6 \mathrm{~mm}$ I.D., Agilent, USA) through a $12.5 \mathrm{~mm} \times 4.6 \mathrm{~mm}$ Zorbax SB-C18 precolumn maintained $20^{\circ} \mathrm{C}$. The mobile phase consisted of methanol- $0.2 \%$ formic acid $(95: 5, \mathrm{v} / \mathrm{v})$. The flow rate throughout the chromatographic analysis was $0.8 \mathrm{~mL} / \mathrm{min}$, and the total run time was 6 minutes. The injection volume was $5 \mu \mathrm{L}$. The retention times were $3.2 \mathrm{~min}$ for bromantane and $5.1 \mathrm{~min}$ for IS.

The detector was operated at unit resolution in the positive MultipleReaction Monitoring-Mode (+MRM) mode using the transitions of the protonated molecular ions of bromantane $(308.1 \rightarrow 135.2 \mathrm{~m} / z)$, IS $(331.0 \rightarrow 250.0 \mathrm{~m} / z)$. MS parameters were optimized by syringe pump infusing of standard solution containing analyte and IS. The spray voltage was set at $2000 \mathrm{~V}$. Corona discharge was $4 \mu \mathrm{A}$. Nitrogen was used as nebulizer gas and pressure was set at 20 psi. Desolvation gas (nitrogen) was heated to $350^{\circ} \mathrm{C}$ and delivered at a flow rate of $5 \mathrm{~L} / \mathrm{min}$. For Collision-Induced Dissociation (CID), high purity nitrogen was used at a pressure of $0.15 \mathrm{MPa}$. During the data acquisition, dwell time was $200 \mathrm{~ms}$. The fragmentation energy of Q1 and collision energy were $135 \mathrm{~V}$ and $25 \mathrm{~V}$ (bromantane), $90 \mathrm{~V}$ and $5 \mathrm{~V}$ (IS), respectively.

\section{Preparation of calibration standards and quality control samples}

A stock solution of bromantane was prepared in methanol at a concentration of $1.0 \mathrm{mg} / \mathrm{mL}$. Standard solutions $(10,25,50,100,500$, $1000,5000 \mathrm{ng} / \mathrm{mL}$ ) were prepared by serial dilution of the stock solution with methanol. Low, medium and high concentration Quality Control (QC) solutions $(4,45,125 \mathrm{ng} / \mathrm{mL})$ were prepared in the similar way. The stock solution of I.S. $(1.0 \mathrm{mg} / \mathrm{mL})$ was also prepared in methanol and then diluted with methanol to a final concentration of $10 \mu \mathrm{g} /$ $\mathrm{mL}$. All solutions were stored at $4^{\circ} \mathrm{C}$ and used within one month after preparation. Calibration standards and QC samples were prepared by adding $50 \mu \mathrm{L}$ standard and $50 \mu \mathrm{L}$ IS or QC solution to $500 \mu \mathrm{L}$ blank human plasma.

\section{Sample preparation}

A sample $(500 \mu \mathrm{L})$ of human plasma was transferred to a $5.0 \mathrm{~mL}$ glass tube, $50 \mu \mathrm{L}$ methanol or $50 \mu \mathrm{L}$ standard of bromantane and $50 \mu \mathrm{L}$ IS solutions were added. If needed (sample not transparent), the mixture was ultra-sonicated. SPE was conducted by using SOLA RP extraction cartridges. The cartridges were conditioned sequentially with $500 \mu \mathrm{L}$ of ethanol and with $500 \mu \mathrm{L}$ of water, and $500 \mu \mathrm{L}$ of plasma sample diluted equal volume of water was then loaded. The loaded cartridges were washed with $500 \mu \mathrm{L} 5 \%$ ethanol in water, and subsequently the analyte was eluted with ethanol. The elution was then evaporated to dryness under $\mathrm{N} 2$ stream at $50^{\circ} \mathrm{C}$, reconstituted with $200 \mu \mathrm{L}$ of methanol, and vortex mixed for $10 \mathrm{~s}$. Finally, the solution was transferred to the autosampler vials, and $5 \mu \mathrm{L}$ was injected into HPLC/MS/MS system.

\section{Method validation}

The method validation assays were carried out according to the currently accepted US Food and Drug Administration (FDA) bioanalytical method validation guidance [14].

The method's specificity was tested by screening six different batches of healthy human blank plasma. Each blank sample was tested for interference using the proposed extraction procedure and chromatographic/spectroscopic conditions and compared with those obtained with an aqueous solution of the analyte at a concentration near to the LLOQ.

The matrix effect on the ionization of analytes was evaluated by comparing the peak area of analytes resolved in blank sample (the final solution of blank plasma after extraction and reconstitution) with that resolved in mobile phase.

Three different concentration levels of bromantane $(4,45$ and $125 \mathrm{ng} / \mathrm{mL}$ ) were evaluated by analyzing five samples at each level. The blank plasma used in this study was five different batches of healthy human blank plasma. If the ratio $<85$ or $>115 \%$, an exogenous matrix effect was implied.

For the calibration standards, peak area ratios (the analyte/IS) were plotted against nominal plasma concentrations, and fitted by weighted $\left(1 / y^{2}\right)$ least-squares linear regression. Plasma calibration curves were prepared and assayed in triplicate on three separate days. In addition, blank plasma was also analyzed to confirm absence of interferences.

The intra-day precision and accuracy of the assay was measured by analyzing five spiked samples of bromantane at each QC level $(4,45$ and $125 \mathrm{ng} / \mathrm{mL}$ ). The inter-day precision and accuracy was determined over three consecutive days by analyzing 15 QC samples. The acceptance criteria for precision and accuracy deviation values should be within $15 \%$ of the actual values. The extraction yield (or absolute recovery) was determined by comparing the bromantane/IS peak area ratios obtained following the outlined extraction procedure (the procedure was a little different from the outlined extraction procedure for QC, calibration curve and clinical plasma samples, that is IS was added to the organic layer after the extraction of bromantane) and the result compared with those obtained from samples which contained the same amount of bromantane in extracted plasma but not be extracted after addition of the drug. This procedure was repeated for the three different concentrations of bromantane added, namely 4,45 and $125 \mathrm{ng} / \mathrm{mL}$.

For sensitivity determination, the LLOQ was defined as the lowest concentration in the calibration curve at which both precision and accuracy were less than or equal to $20 \%$, and signal/noise $(\mathrm{S} / \mathrm{N})>10$. The $1 \mathrm{ng} / \mathrm{mL}$ concentration was investigated as the lower limit of quantification. Reproducibility and precision were also determined.

To evaluate stability on repeat analysis of samples, freeze-thaw stability was determined for three concentrations of bromantane in plasma. QC plasma samples were tested after three freeze $\left(-20^{\circ} \mathrm{C}\right)$ and thaw (room temperature) cycles.

\section{Results and Discussion}

\section{LC/MS/MS conditions}

Certain classes of compounds are traditionally very difficult to ionize or tend to show low sensitivity in LC/MS/MS techniques. Although electrospray ionization is often used as an LC-MS interface, APCI, another type of atmospheric pressure ionization, is more applicable to low polarity compounds [15]. In APCI, the corona discharge can provide a source of electrons in the gas-phase. This can be an advantage for such non polar compounds as adamantanes.

The LC-MS/MS operation parameters for determination of bromantane and IS were carefully optimized. Selenox was selected as the IS because its chromatographic behavior and extraction efficiency were similar to those of analyte. Both analyte and IS responded best to the positive ionization mode, with the protonated molecular ions $[\mathrm{M}+\mathrm{H}]^{+}$as the major species. Full scan ion spectrum of Bromantane was shown in figure 1. The MRM acquisitions were performed at unit resolution using the transition $308.1 \rightarrow 135.2 \mathrm{~m} / \mathrm{z}$ for bromantane and $331.0 \rightarrow 250.0 \mathrm{~m} / \mathrm{z}$ for IS respectively (Figure 2). The transition $308.1 \rightarrow 135.2 \mathrm{~m} / z$ was used for quantification of bromantane because of its stabilized ions response 


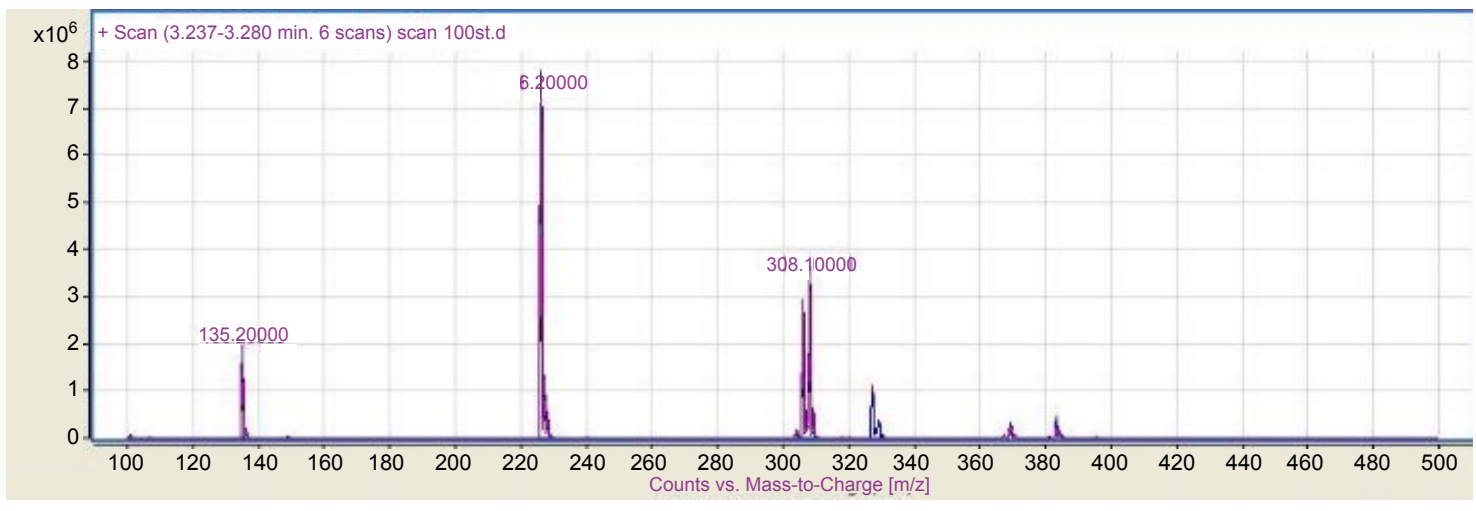

Figure 1: Full scan positive ion turbolonspray Q1 mass spectrum of Bromantane.

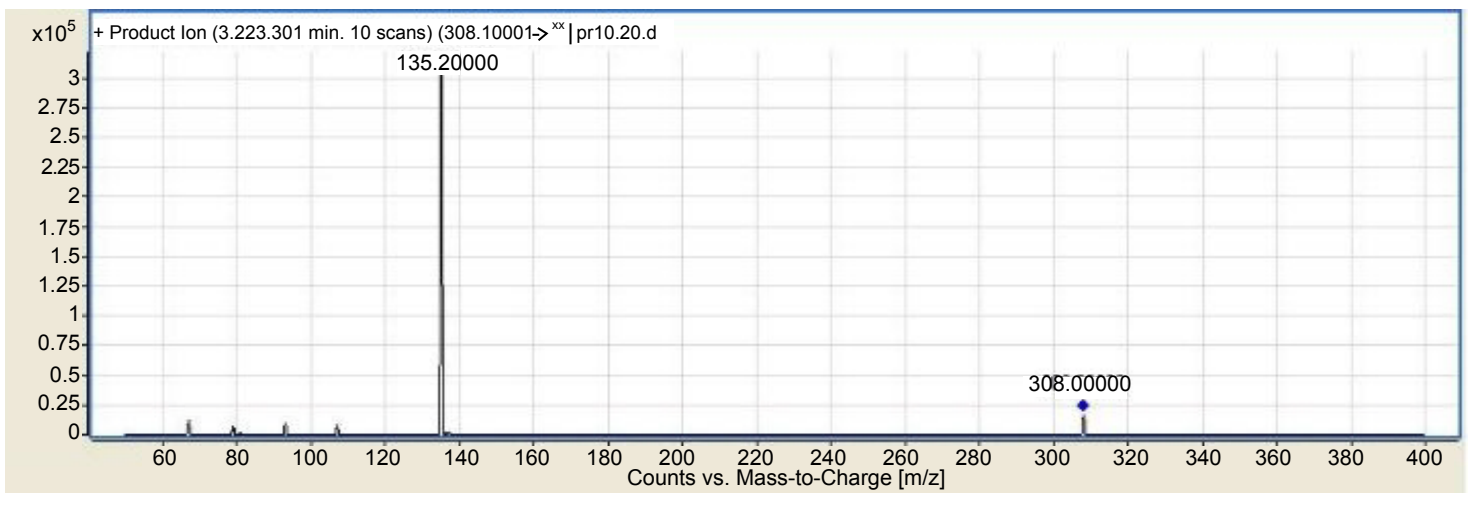

Figure 2: $M S / M S$ spectrum of the $\mathrm{m} / \mathrm{z}=308.1$ parent ion, exhibiting the product ions of Bromantane at $\mathrm{m} / \mathrm{z}=135.2$.

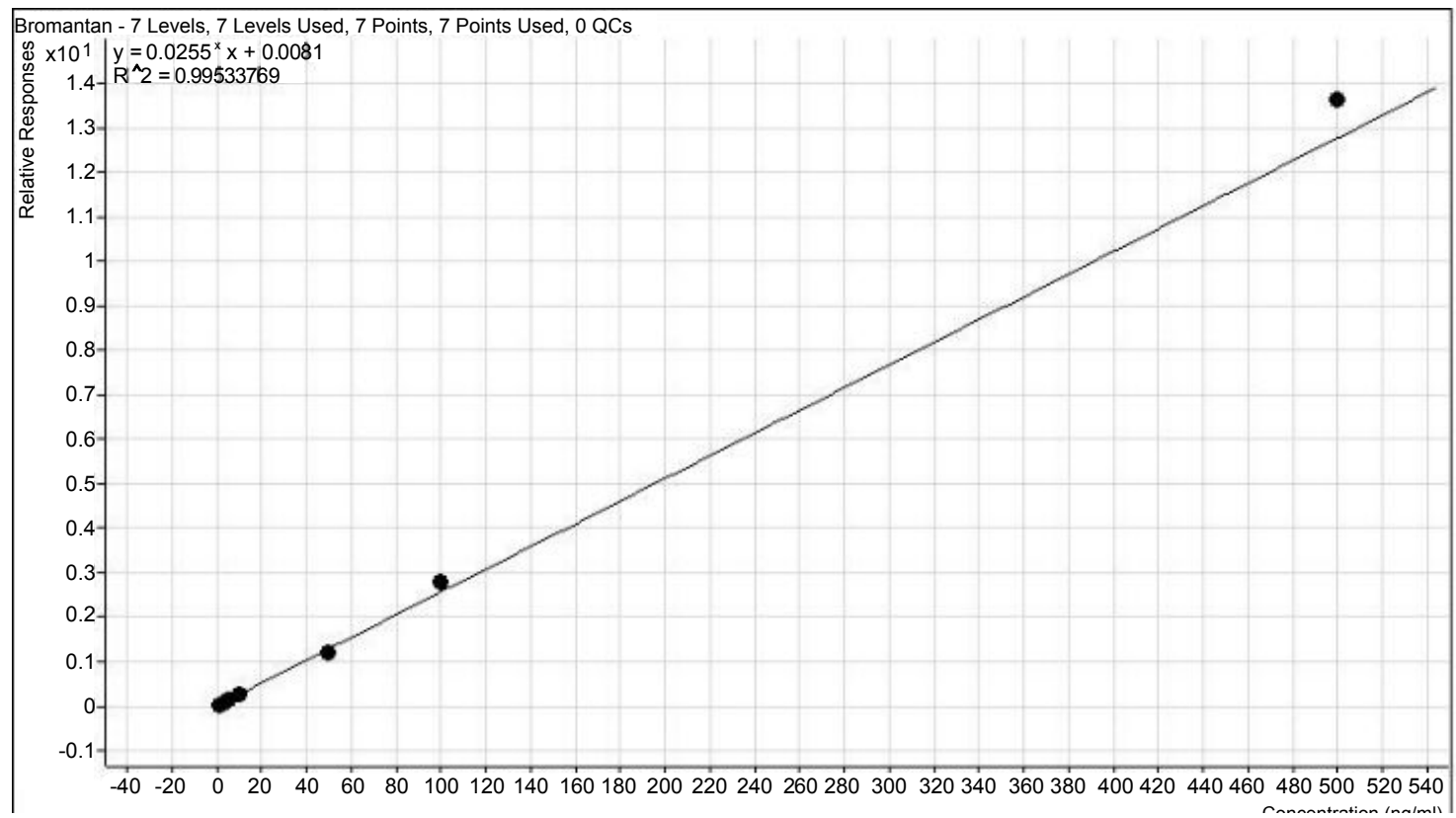

Figure 3: Regression line for the determination of Bromantane in human plasma.

and no endogenous interference. The mass parameters were optimized by observing the maximum response obtained for the product ions. The intensities of its protonated molecules were compared at fragmentor voltages of $90,100,120,130,135,140$ and $150 \mathrm{~V}$ in order to determine the optimal collision energy. The results showed that the highest sensitivities could be obtained by using a $135 \mathrm{~V}$ fragmentor voltage. 
Furthermore, the mobile phase system was optimized through several trials to achieve satisfactory chromatographic behavior and the ionization responses of bromantane and IS. The ionization of bromantane and IS was affected by the composition of mobile phase. In the preliminary experiments, methanol, acetonitrile, ammonium acetate and formic acid in various proportions were tested. Methanol, rather than acetonitrile, was chosen as the organic modifier because it leads to the good peak shape of bromantane and satisfactory retention time. When ammonium acetate was added in the mobile phase, the response of the analyte was distinctly decreased. Both the analyte and IS were found to have higher response and better peak shapes in the mobile phase containing $0.5 \%$ formic acid. Finally, in terms of peak shape, retention time and sensitivity, we employed methanol $-0.5 \%$ formic acid $(95: 5, \mathrm{v} / \mathrm{v})$, as the mobile phase.

\section{Assay validation}

The linearity for bromantane was investigated by linear regression of peak area ratios against concentrations (Figure 3). The regression equation for the calibration plot was $y=0.081+0.0255^{\star} \mathrm{x}$, with a coefficient of correlation $(r)$ of 0.995 (where y - the ratio of the peak areas of bromantane to the peak area of the internal standard; $\mathrm{x}$ - bromantane concentration, $\mathrm{ng} / \mathrm{mL}$ ). The calibration curves of bromantane showed good linearity in the ranges of $1-500 \mathrm{ng} / \mathrm{mL}$ (Table 1). LLOQ for bromantane in plasma was proved to be $1 \mathrm{ng} / \mathrm{mL}$ $(\mathrm{S} / \mathrm{N}=9.5)$ (Figure 4).

Our investigations have confirmed the fact of negligible matrix effects coupled to APCI [16]: the ratios of the peak responses for bromantane were $99.0 \%, 98.9 \%$, and $100.3 \%$ at 4,45 and $135 \mathrm{ng} /$ $\mathrm{mL}$, respectively (Figure 5). The results indicated that co-eluting endogenous substances hardly inhibited the ionization of bromantane, and the ion suppression from human plasma matrix was consistent for this analytical method and would not interfere with the measurement of analyte. Thus, no ion suppression was observed.

The extraction recovery determined for bromantane was shown to be consistent, precise and reproducible. The absolute recoveries of bromantane from the rat plasma at the three concentrations were about $89 \%$. There was no significant difference in the extraction efficacy of the present assay over the range of concentrations studied.

The intra-day and inter-day precision $(\mathrm{CV}, n=5)$ for bromantane were satisfactory at the three concentrations studied. Data on precision, accuracy are shown in table 2. The results of freeze-thaw stability indicated that the analyte is stable in plasma for three freeze-thaw cycles, when stored at $-20^{\circ} \mathrm{C}$ and thawed to room temperature. Long-term stability indicates that storage of bromantane plasma samples at $-20^{\circ} \mathrm{C}$ is adequate when stored for 30 days and no stability related problems would be expected during routine analysis for the pharmacokinetic or abuse control studies.

\section{Conclusion}

The proposed method of analysis provided a sensitive and specific assay for bromantane determination in human plasma. No significant interference caused by endogenous compounds was observed. The method was rapid, selective and highly sensitive with a LLOQ of $1 \mathrm{ng} /$ $\mathrm{mL}$ for bromantane. Simple liquid-liquid extraction procedure and short run time can provide a short analysis time that is important for large sample batches. The developed method was completely validated showing satisfactory data for all the method validation parameters

\begin{tabular}{|c|c|c|c|c|c|c|}
\hline Spike Conc. & RT & Final Conc. & Accuracy & Area & $S / N$ & Area IS \\
\hline 1 & 3.21 & 1.02 & 102.3 & 661 & 9.5 & 19337 \\
\hline 2.5 & 3.22 & 2.53 & 101.4 & 1321 & 16.0 & 18147 \\
\hline 5 & 3.22 & 4.67 & 93.4 & 2305 & 26.7 & 18105 \\
\hline 10 & 3.22 & 9.63 & 96.3 & 3841 & 16.3 & 15123 \\
\hline 50 & 3.22 & 47.52 & 95.0 & 19068 & 82.6 & 15611 \\
\hline 100 & 3.22 & 109.02 & 109.0 & 44764 & 143.2 & 16035 \\
\hline 500 & 3.22 & 533.34 & 106.7 & 246547 & 209.4 & 18096 \\
\hline
\end{tabular}

Table 1: The linearity of Bromantane determination.

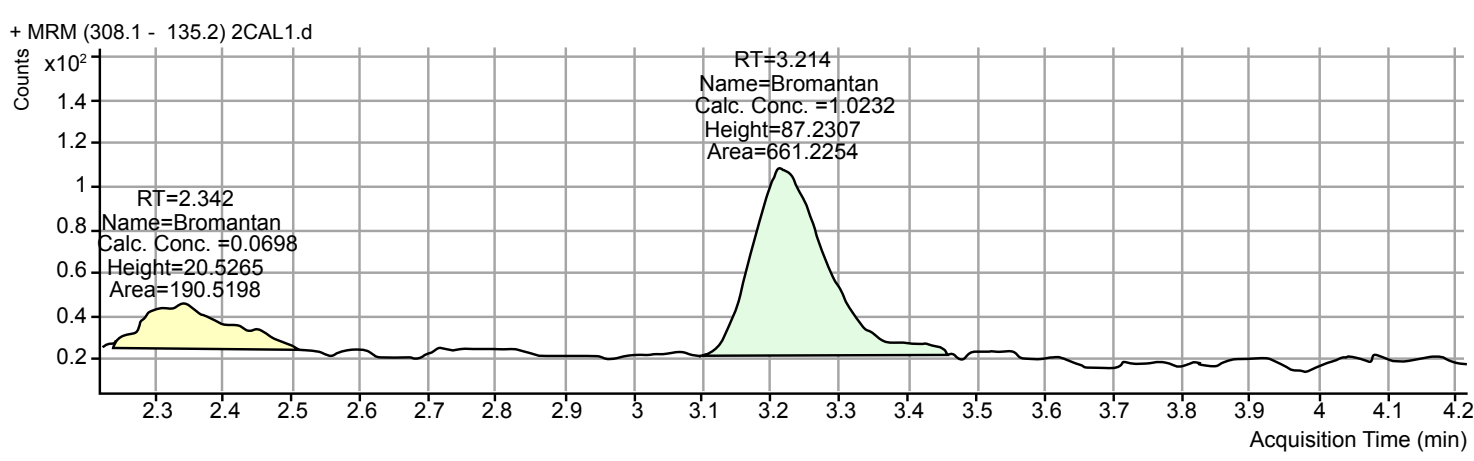

Figure 4: MRM mass chromatograms of LLOQ (1 ng/mL) of Bromantane.

\begin{tabular}{|c|c|c|c|c|}
\hline Calculated $\boldsymbol{C}(\mathbf{n g} / \mathbf{m l})$ & Found $\boldsymbol{C}(\mathbf{n g} / \mathbf{m l})$ & Intra-day precision R.S.D. (\%) & Inter-day precision R.S.D. (\%) & Accuracy RE (\%) \\
\hline 4 & 4.26 & 7.5 & 6.6 \\
\hline 45 & 44.26 & 9.4 & 8.9 \\
\hline 135 & 138 & 5.1 & -1.6 \\
\hline
\end{tabular}

Table 2: Accuracy and precision of the assay for determination of Bromantane in plasma $(n=5)$. 


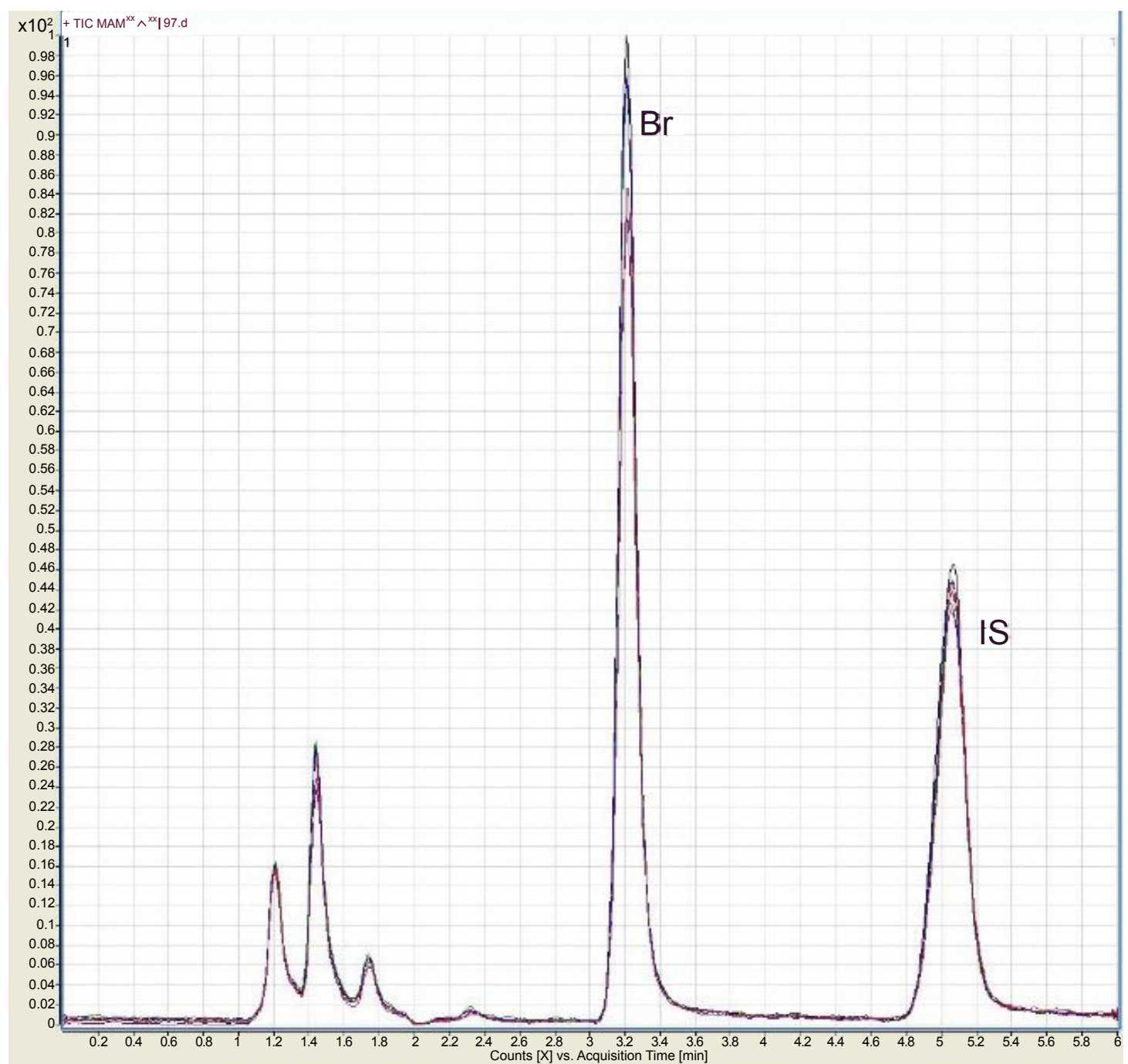

Figure 5: Typical mass chromatograms of plasma spiked with Bromantane (45 ng/mL) and the IS (1 $\mu \mathrm{g} / \mathrm{mL})$, validation of Bromantane Quantitation

tested. It was shown that this method is suitable for the analysis of bromantane in plasma samples collected for pharmacokinetic, bioavailability, bioequivalence studies or doping control.

\section{Acknowledgments}

The authors are thankful to prof. N.N. Zolotov from the Institute of Pharmacology Russian Academy of Medical Sciences for providing sample of Bromantane for the research work.

\section{References}

1. Krapivin SV, Sergeeva SA, Morozov IS (1993) A quantitative pharmacoelectroencephalographic analysis of the action of bromantane. Biull Eksper Biolog Med 116: 515-518.

2. Grekhova TV, Gaĭnetdinov RR, Sotnikova TD, Krasnykh LM, Kudrin VS, et al. (1995) The effect of bromantane, a new immunostimulant with psychostimulating action, on release and metabolism of dopamine in the dorsal striatum of freely moving rats: a microdialysis study. Biull Eksper Biol Med 119: 302-304

3. Kudrin VS, Sergeeva SA, Krasnykh LM, Miroshnichenko II, Grekhova TV, et al. (1995) The effect of bromantane on the dopamin- and serotoninergic systems of the rat brain. Eksper Klin Farmak 58: 8-11.

4. Morozov IS, Petrov V I, Sergeeva SA (2001) The Pharmacology of Adamantanes. Volgograd State Medical Academy, Volgograd.
5. Burnat P, Payen A, Le Brumant-Payen C, Hugon M, Ceppa F (1997) Bromontan, a new doping agent. Lancet 350: 963-964

6. Bowers LD (2009) The analytical chemistry of drug monitoring in athletes. Annu Rev Anal Chem 2: 485-507.

7. Morozov IS, Pukhova GS, Avdulov NA, Sergeeva SA, Spasov AA, et al. (1999) The mechanisms of the neurotropic action of bromantan. Eksper Klin Farmak 62: $11-14$

8. Gui K, Wu M, Liu X, Zhang Y, Wang S (1999) Study on the metabolites of bromantane in urine using GC/MS and GC/Tandem MS/MS. In: Schanzer W, Geyer H, Gotzmann A, Mareck-Engelke U (Eds.), Recent advances in doping analysis. Sport and Buch Straus, Koln: 375-390.

9. Niwa M (2012) Chemical derivatization as a tool for optimizing MS response in sensitive LC-MS/MS bioanalysis and its role in pharmacokinetic studies. Bioanalysis 4: 213-220

10. Arndt T, Guessregen B, Hohl A, Reis J (2005) Determination of serum amantadine by liquid chromatography-tandem mass spectrometry. Clin Chim Acta 359: 125-131.

11. Almeida AA, Campos DR, Bernasconi G, Calafatti S, Barros FA, et al. (2007) Determination of memantine in human plasma by liquid chromatographyelectrospray tandem mass spectrometry: application to a bioequivalence study. J Chromatogr B 848: 311-316. 
Citation: Miroshnichenko II, Sergeeva SA, Platova AI, Krasnykh LM (2013) A Rapid and Sensitive LC-MS/MS Assay for the Quantitation of Bromantane in Human Plasma. J Sports Med Doping Stud 3: 120. doi:10.4172/2161-0673.1000120

12. Konda RK, Challa BR, Chandu BR, Chandrasekhar KB (2012) Bioanalytical method development and validation of memantine in human plasma by high performance liquid chromatography with tandem mass spectrometry: application to bioequivalence study. J Anal Methods Chem 2012: 1-8

13. Meadows K, Liddicoat T, Oliver M (2012) Revolutionizing SPE for improved bioanalysis. Bioanalysis 4: 2661-2663.

14. Guidance for Industry, Bioanalytical Method Validation (2001) US Department of Health and Human Services, Food and Drug Administration, Center for Drug Evaluation and Research (CDER)

15. Hourani N, Kuhnert N (2012) High molecular weight non-polar hydrocarbons as pure model substances and in motor oil samples can be ionized without fragmentation by atmospheric pressure chemical ionization mass spectrometry. Rapid Commun Mass Spectrom 26: 2365-2371.

16. Ackermann BL, Berna MJ, Eckstein JA, Ott LW, Chaudhary AK (2008) Current applications of liquid chromatography/mass spectrometry in pharmaceutical discovery after a decade of innovation. Annu Rev Anal Chem 1: 357-396. 